\title{
Communications
}

2019; 7(2): 31-39

http://www.sciencepublishinggroup.com/j/com

doi: 10.11648/j.com.20190702.11

ISSN: 2328-5966 (Print); ISSN: 2328-5923 (Online)

\section{Practical Development of Creative Life-Saving Artificial Intelligence}

\author{
Evgeniy Bryndin \\ Research Department, Research Center "Natural Informatics", Novosibirsk, Russia
}

\section{Email address:}

bryndin15@yandex.ru

\section{To cite this article:}

Evgeniy Bryndin. Practical Development of Creative Life-Saving Artificial Intelligence. Communications. Vol. 7, No. 2, 2019, pp. 31-39. doi: 10.11648/j.com.20190702.11

Received: November 8, 2019; Accepted: November 23, 2019; Published: December 2, 2019

\begin{abstract}
Artificial intelligence is a revolutionary technology that is designed to transform the life of the world community: to optimize business processes, to provide valuable information, to increase creative service to citizens. Both technological and cultural changes are required to realize the maximum capabilities of artificial intelligence. It needs to be implemented in all components of the business, and all departments and employees need to be given the opportunity to integrate artificial intelligence into their daily tasks. Such a fundamental change in corporate culture should begin with the leadership of the organization. This requires investment not only in technical infrastructure, but also in training staff so that all employees can derive the maximum possible benefits from artificial intelligence. In addition to changing culture, new generation technologies, such as a neural processor, are needed to develop artificial intelligence, which will allow the use of machine learning to obtain useful analytical information from data. Neural processors are able to fundamentally transform different industries through the application of artificial intelligence. This will allow firms to increase the creativity of their services. The importance of integrating artificial intelligence into the infrastructure of the future has already been recognized. The Government AI Readiness Index has been created, which reflects the readiness of Governments to support the development of artificial intelligence technology. The article discusses the main aspects of the formation of creative life-saving strong artificial intelligence.
\end{abstract}

Keywords: Creative Artificial Intelligence, Systems and Methods, Scientific Modeling, Creative Technology

\section{Introduction}

Creativity has traditionally been considered to belong exclusively to man. A person's creative intelligence is formed on the basis of feeling - knowledge. The feelings of a person with acquired skills learn and accumulate professional experience and competence. Based on skills and experience, a person begins to feel new professional situations. This helps him acquire new professional skills. Reason conceptually defines them in the form of knowledge. A person begins to accumulate new professional experience.

Artificial intelligence was capable of creativity. Artificial intelligence can accumulate experience in the process of modeling professional activities. Based on the experience gained, artificial intelligence can also improve and develop models of different professions and shape the model and technology of new professional activities. So artificial intelligence becomes creative [1-16].
Creative life-saving strong artificial intelligence is a digital twin of human intelligence, capable of learning, retraining, self-realization and development by improving functional activities by mastering creative innovative high-tech professional and behavioural skills and competences according to criteria of preferences with qualitative choices.

Neural networks have learned to paint paintings, compose music and poems, and invent scripts for films. Yandex teaches neural networks to record music albums similar to those of popular bands and write poems. The principle of operation of all algorithms is similar: they analyze a huge array of works of art, and then on the basis of the obtained patterns create their creation: The PIX18 algorithm invented by Creative Machines Lab can generate works based on photos at its disposal. Paintings created by Google 's DeepDream algorithm are considered practically art. Neural networks can even create a new one. Developers from the Laboratory of Artificial Intelligence and Art of Ratger University, created a generative- 
adversarial network GAN of two discriminators. An algorithm based on the answers of one discriminator analyzed the paintings, drew its own and checked the result. He produced images similar to those he had studied before. The second discriminator, analyzes about 81 thousand paintings and, based on such a voluminous sample, forms a list of conditions under which the created painting can be classified as works of art. At the same time, the second discriminator makes a list of styles and checks the picture for similarity with them - carries out a verification operation. A new painting is born when the image is recognized as a work of art not identical to any of the preexisting styles. The GAN network follows creativity, produces new paintings that provoke emotional response in people.

Project Alejandro Giacometti of EDITED, an analytics company for the retail. During the experiment, he recognized thousands of images from the podiums of the spring show of London Fashion Week in 2017, which allowed to allocate to the photo the image of individual parts of clothing. For this purpose, a system of two neural networks was used: the first subnet formed a unique representation of the input image in the form of a set of real numbers, and the second restored the original image from it. In the course of training, the parameters of these subnets were selected so that the obtained picture was as similar as possible to the original. Thus, the first subnet was able to learn to obtain compact information from the visual image of clothing, which became possible to analyze - for example, to assess the similarity of two outfits among themselves. The second subnet made it possible to create a "digital model" of outfits, describing dozens of parameters of each. When researchers accumulated enough data, they were able to teach the computer to distinguish the models of one designer from others, to find characteristic elements of style. Moreover, it became possible to predict the emergence of new collections, to predict consumer demand.

Google, along with the German company Zalando SE, have created a neural network that helps designers determine which styles and clothes will suit certain people and be in demand. For consumers, the technology helps to select clothes based on responses to the questionnaire and test drawing.

The service "Ivanex Autopate," which was created in December 2013, has been making poem lines based on requests of users and news headlines for several years. For this purpose, the robot was specially taught to determine poem dimensions and rhyme lines among themselves.

Employees of the Moscow Automobile and Road Technical University have developed artificial intelligence, which has multifunctional abilities: manages different modes of ground transport, aircraft, solves complex geometric tasks, knows how to build long logical chains.

Today, in addition to independent "production" of poems, paintings and music, neural networks help scientists to carry out research in the creative sphere. The development of the modern music industry focuses on classified patterns that help literally build a mathematical model of music composing.
American psychologist Colin Martindale highlighted two stages of the cognitive process. The primary process is nondirectional, irrational thinking. The secondary process is conscious, conceptual, it is the solution of specific tasks and the use of logic. He also attached this to the creative process. Artificial intelligence has no irrational thinking. Neural networks can write poems, but it is our perception, our reading, that fills them with meaning. For the algorithm, words, smears, colors and sounds are just a set of signs it can fold into a harmonious structure. Artificial intelligence does not see the content, the sense field. It cannot give objects meaning and works are of global cultural value. Artificial intelligence can create a brilliant symphony or a combination of rhymes, properly organized graphically, but only recognition of man will allow all this to gain so much desired by many - really to be art, not to appear to be. And although artificial intelligence will not replace human imagination, in the future this technology will become an important tool for revealing creative potential.

In the international professional community, creative lifesaving strong artificial intelligence becomes almost relevant. It's evolving from big data.

\section{Data Attributes}

Objects, objects, materials, things, processes, phenomena and other aspects of the physical world have different properties.

Properties are represented by qualitative content attributes.

A qualitative attribute can be visual or audible. A meaningful attribute can be represented by a number, a language sense value, a visual or audible way, a mathematical or behavioral action, or an algorithm.

Meaningful qualitative attributes are artificial intelligence data.

Big Data - the data collected automatically is too large for manual collection and processing to find new patterns and knowledge that cannot be derived from local data fragments

Big Data attributes of the fields of economics, industrial industries, technology and professions help build and train artificial neural multilayer artificial intelligence networks to manage, make decisions and issue recommendations to professionals and executives.

Applied research modeling helps accumulate Big Data scientific attributes in real time and simultaneously use them to deeply train multi-layer artificial neural control networks to simulate applied research, make decisions, and make recommendations to researchers.

Mathematical methods, cognitive systems, and technology are used to process big data.

\section{Artificial Intelligence Methods, Systems and Techniques}

Recommendation Systems (Collaborative Filtering) are systems that provide recommendations based on implicit 
patterns detected by AI algorithms using big data analysis. They can be aimed at interaction with the client (offer goods/services/dating), or used within companies (to support credit decisions, personnel decisions).

Predictive systems are systems that capture relationships between variables in historical data sets and their outcomes. Based on these relationships, models are developed, which in turn are used to predict new results.

Biometrics - removal and digitization of various biological characteristics of a person - faces, gestures, gait, fingerprints, blood pressure, temperature, etc. Used for identification of people and human-machine interactions.

Knowledge Management is the representation of knowledge about the world in the form of a hierarchy of concepts, such that the AI system can use knowledge to solve complex problems such as disease diagnosis, decision making, or human conversation.

Man-Machine Interface. Person-Device Dialog - A dialog (text or voice) in a client or technical support service. Entertainment robots. Dialogue with household appliances, car, disabled.

Virtual interlocutors (chatbota, Chatbots) - the programs communicating with the person in a natural language. Can work as promoters, account managers, help human resources, answer frequent questions in online technical support services, use in cars and home entertainment robots. Communication can be text (chat-like) or voice.

Machine Learning (ML) is an AI area that creates algorithms that independently learn and predict the situation based on available data without requiring the intervention of a programmer.

Supervised Learning is a type of machine learning where the learning algorithm is given pre-classified and sorted data consisting of examples of input data and desired results of its processing. The purpose of the training is to learn the general rules that link input and results and, based on these rules, to predict future events.

Unsupervised Learning is a kind of machine learning in which the learning algorithm does not include any classifications and labels, itself defines the structure and relationships of the input data. Uncontrolled learning can itself be a goal (detection of hidden patterns in data) or a means (extraction of features from an array of data). It is focused on the investigation of input data and recognition of the structure of untagged data.

Reinforcement Learning is a type of machine learning in which an algorithm has a specific purpose - for example, to control a robotic manipulator or play Go. Every step on the way to the goal is marked by a reward or a fine. Given this feedback, the algorithm can work out the most efficient path to the target.

Deep Neural Networks is a multi-layer neural network in which "learned" data is passed through several layers of "neurons"; On each layer, the result of the previous layer is used as input information.

Convolutional Neural Networks. Multilayer neural networks with alternation of special curving (summing) and ordinary layers, most effective for image recognition.

Feed-Forward Networks is a neural network with many layers where data is propagated only forward.

Recurent Neural Networks are deep neural networks where data can propagate between layers forward and backward.

Neural Network Constructors/Libraries

(Frameworks/Neural Frameworks, Deep Learning Frameworks). Neural network development environment "under task," allowing generate several dozen different types of neural networks and then train them on given datasets. There are only a few popular neural libraries, the main players of this market - TensorFlow (Google), PyTorch (Facebook), Keras (open-source), etc.

Graphics accelerators (Graphics Processor Unit (GPU)). Graphic maps for displaying images on the computer screen, allowing to carry out ultra-fast and massive parallel calculations, due to this became the main carrier of calculations on neural networks. The main player of this market is Nvidia.

Neural chips (Neural Processing Unit, Neuromorphic Processor, Tensor Processing Unit, ASIC). Highly specialized processors specifically optimized for fast parallel computing using neural networks.

Dataset (Training Set) - a set of precedents (objects, cases, events, subjects, texts, photographs, samples, etc.) selected to train an algorithm from a variety of all possible precedents, called a general set.

Mark-up. Processing a learning sample to assign properties to objects in it that the algorithm is learning to recognize.

Marking system. An automated workplace that allows many machine learning operators to quickly map learning samples. For example, manual allocation of persons by the operator in photos, marking of letters on spam/non-spam, marking of search results or X-rays.

Open data - archives, libraries of data, usually stored in cloud storage and available for download anywhere in the world to anyone.

Data Processing Center, Data Center - a special technical room, which houses special equipment (servers, server racks, network equipment, etc.) for processing, storage and transmission of large data arrays, with power supply and powerful communication channels. Modern data centers are complex engineering facilities and consume a large amount of electricity (up to hundreds of megawatts), most of which goes to cooling the equipment.

The data set (data set) represents a set of the data having identical structure and united on sense. The dataset can be both text, sound, visual and tabular information and all kinds of combinations thereof. The dataset may contain data annotated (marked) or unannotated (unmarked).

Tagged data - containing structured information for all dataset instances that include meta-information to solve specific applications. The markup shall be unified, have the property of integrity and meet the requirements of meta content.

Julia is a high-level general-purpose programming language developed in 2009 with a focus on high- 
performance numerical analysis and computational science. Simple syntax and deep mathematical roots make Julia a friendly programming language for data analysts, offering the perfect way to express algorithms. It also includes Flux, the basis for machine learning and supports TensorFlow and MXNet.

Python is a widely used programming language and can be used to implement AI because of the simple and seamless structure it offers. Python syntax makes it easy to implement different AI algorithms, which also reduces development time compared to other available programming languages. Applying Python allows users to create neural networks with a set of useful libraries that can be used to develop AI. Other features include the ability to test algorithms without having to implement them.

Java, an extremely popular cross-platform programming language, provides search algorithms and neural networks. It is an easy-to-understand language that offers graphical representation, debugging, and scalability. Its portability makes it the preferred implementation for different applications based on having different built-in types.

Tentorflow is an open software library for machine learning developed by Google to solve the problems of building and training a neural network with the aim of automatically finding and classifying images, achieving the quality of human perception. It is used for both research and development of Google 's own products. The main API for working with the library is implemented for Python, and there are also implementations for C, Haskell, Java, Go, and Swift. Continues a closed DistBelief project. Originally TensorFlow by the Google Brain team for internal use at Google, in 2015 the system was transferred to free access with an open license Apache 2.0. TensorFlow is well suited for automated image annotation in systems such as DeepDream. TensorFlow allows training of GANs.

PyTorch is an open-source Python machine learning library built on Torch. Used for natural language processing. It is developed predominantly by Facebook 's artificial intelligence group. Uber 's "Pyro" library for probabilistic programming is based on PyTorch. PyTorch provides two main high-level models: 1. Tensor computing (similar to NumPy) with advanced acceleration support on GPU, 2. Deep neural networks based on autodiff system.

Keras is an Open Neural Network Library written in Python. It is an add-on above the Deeplearning4j, TensorFlow and Theano frames. It is aimed at operational work with deep learning networks, while being designed to be compact, modular and expandable. According to the concept, Keras is an interface rather than an end-to-end machine learning system. Keras provides a high-level, more intuitive set of abstractions that makes it easy to form neural networks, regardless of the scientific computing library used as a computational backend. Microsoft is working on adding CNTK libraries to Keras and low-level libraries.

Caffe is the foundation of deep learning, created with speed and modularity in mind. Switching between the central and graphic processing unit on the computer, and then expansion on computing clusters or mobile ustroi stvo. Speed makes Caffe ideal for scientific experiments and industry. Caffe can process 60 million the image in day with one GPU NVIDIA.

CUDA is a parallel hardware and software architecture that significantly improves computing performance with Nvidia graphics processors. The CUDA SDK allows programmers to implement algorithms in special simplified dialects of the $\mathrm{C}$, $\mathrm{C}$ and Fortran programming languages that are feasible on Nvidia graphics and tensor processors. The CUDA architecture gives the developer the option, at his own discretion, to organize access to the set of instructions of the graphic or tensor accelerator and to manage its memory. Functions accelerated with CUDA can be called from a variety of languages, including Python, MATLAB, etc.

Clouds - computing services (servers, storage, network, software) accessible via the Internet. In fact, you can buy computing power or storage without thinking about what servers and where they are - somewhere "in the cloud." For example, to avoid storing personal documents and photos on a personal computer's hard drive, most users now store them online.

\section{Creative Artificial Intelligence of Applied Direction}

The Russian company "Big Troika" has developed a software and hardware complex (SHC) "Waste Management," which is engaged in forecasting and mathematical modeling of the situation in the waste management industry. It is based on a mathematical model that uses optimization algorithms and works with big data. As result, SHC can fully cover the waste management industry not only in individual regions, but also in the country as a whole, while responding to the needs of all its participants. The Waste Management Complex can calculate the current needs of the region for waste management and develop a complete scheme for the development of the industry, providing for the need for modern infrastructure facilities: waste sorting and processing plants, waste handling stations, landfills that exclude soil pollution and other facilities that require long-term investment. Such a scheme takes into account the economic and logistical factors characteristic of each region and suggests which solutions will be most effective. With the help of the system it is also possible to calculate how the waste management industry in the region will work, and to predict tariffs for 10-15 years ahead. You can see how any changes will affect the situation: the decommissioning of old or the construction of new waste management facilities, the use of different processing technologies, export routes, the choice of location for the new facility. You can find out how much each such solution will cost the consumer, and therefore find the optimal and economically viable solution for all participants.

One of the key tasks in the development of the territorial scheme is the collection and processing of information. It is 
necessary to obtain reliable data on all market participants on waste management as soon as possible. These are tens and hundreds of thousands of legal entities, waste collector companies, hundreds of waste transport companies from thousands of vehicles, tens of thousands of container sites. Errors at this stage of data collection can lead to the construction of an incorrect territorial scheme for waste management. For this purpose, the Big Three electronic models already have synchronization with federal, regional and commercial information resources. The real-time control system processes a huge amount of data and constantly trains our mathematical models so that they in turn build forecasts in electronic models. Thus, models are constantly adjusted to work even more accurately. As a result, this allows to reduce tariffs for garbage collection.

SHC Waste Management consists of three modules, each of which performs certain tasks. For example, the Regional Waste Inventory Automated Information System (AIS) allows the collection and processing of data on those who create garbage: apartment buildings, individual residential buildings and legal entities, such as restaurants or cafes. The system employs waste management industry operators and representatives of state authorities who control reporting. The system automatically fills out forms and issues fines for detected violations of the law, as well as shows up-to-date and correct data on waste management in the region. The second module, the "Territorial Scheme Editor," takes into account up-to-date information on all objects that participate in the waste management industry in the region: garbage containers and polygons, garbage trucks, incinerators and sorting stations. The mathematical algorithm allows to route the garbage truck in such a way that garbage collection takes place more efficiently, as well as to calculate how to load infrastructure objects so that they work longer. Given many factors, AIS is also able to identify the most appropriate location for the construction of new facilities and predict when such a need arises. The third module of the software and hardware complex is the automated system "Waste Management," a tool to support the business processes of the regional operator or management company at the landfill or sorting yard. From contracting to assigning daily tasks to garbage truck drivers - the system covers all operator activities, automatically laying and optimizing routes, as well as taking into account the amount of waste at infrastructure facilities. Digitalization also touched the drivers of garbage trucks: daily tasks they receive with the help of a special mobile application, pre-installed on strong and waterproof tablets. Performance reports are prepared automatically, and equipment installed on containers and cars allows real-time monitoring of progress.

Physicists at Lawrence National Laboratory in Berkeley created a machine learning algorithm that improved synchrotron radiation characteristics. Physicists created a neural network into which ALS synchrotron operation data was loaded. The neural network recognized patterns in this data and determined how different parameters of the device affect the width of the electron beam. The machine learning algorithm also recommended adjusting the position of magnets to optimize the final beam. The ALS-U project increased the focusing of light beams from about $100 \mu \mathrm{m}$ to less than $10 \mu \mathrm{m}$.

American physicists have applied machine learning algorithms to study the phases of a quantum particle system. The study of quantum systems of many bodies presents a very difficult computational task, as with the addition of each new particle its complexity grows exponentially. Physicists used machine learning to construct a phase diagram for the case of 12 particles. The neural network was able to indicate the boundaries between phases more accurately than any approach used before.

A group of scientists led by Anton Zailinger of the Institute of Quantum Optics and Information of the University of Vienna, created a software algorithm that allows the development and planning of new experiments in the field of quantum physics. The algorithm, called Melvin, runs variants of experimental components and is able to learn from its previous successful solutions, which allows to significantly speed up the process of development of more complex experiments. Scientists using this algorithm have already calculated several experiments and received dozens of new results [17]. These results show that already now artificial intelligence is capable of creativity and in the future these abilities will only increase.

Artificial intelligence, which did not contain any knowledge of chemistry, re-opened Mendeleev's table and told scientists new promising materials. For this purpose, he analyzed 3.3 million annotations of scientific works. The achievement is made by a group led by Anubhav Jain of the Lawrence National Laboratory in Berkeley, United States.

Artificial Intelligence and Nanotechnologies

Nanotechnologies focused on materials with new properties are an important area of applied scientific research. Artificial intelligence is able to detect properties of the obtained material from samples in the process of modeling the transformation of nanoprocesses and compare them with the specified properties [18].

Nanoprocesses are characterized by stable ion-electron interaction at the molecular level. Simulation of transformation of nanoprocesses is carried out by nanovisual actions on sample before obtaining material with stable ionelectronic interaction. Evaluation is carried out on the basis of stability criterion of ion-electronic interaction of molecular structure.

The properties are identified by identifying attributes from the real-time big data stream in the simulation process. Cognitive analysis of big data flow and their comparison with attributes of specified properties are carried out. Cognitive analysis of attributes of properties of new materials and comparison of them with attributes of specified properties is carried out by multilayer artificial neural network. The universal scheme of modeling scientific experiments based on strong creative artificial intelligence is published in the article [19] is shown in Case 1.

Case 1. Application of Strong Artificial Intelligence 
Table 1. General.

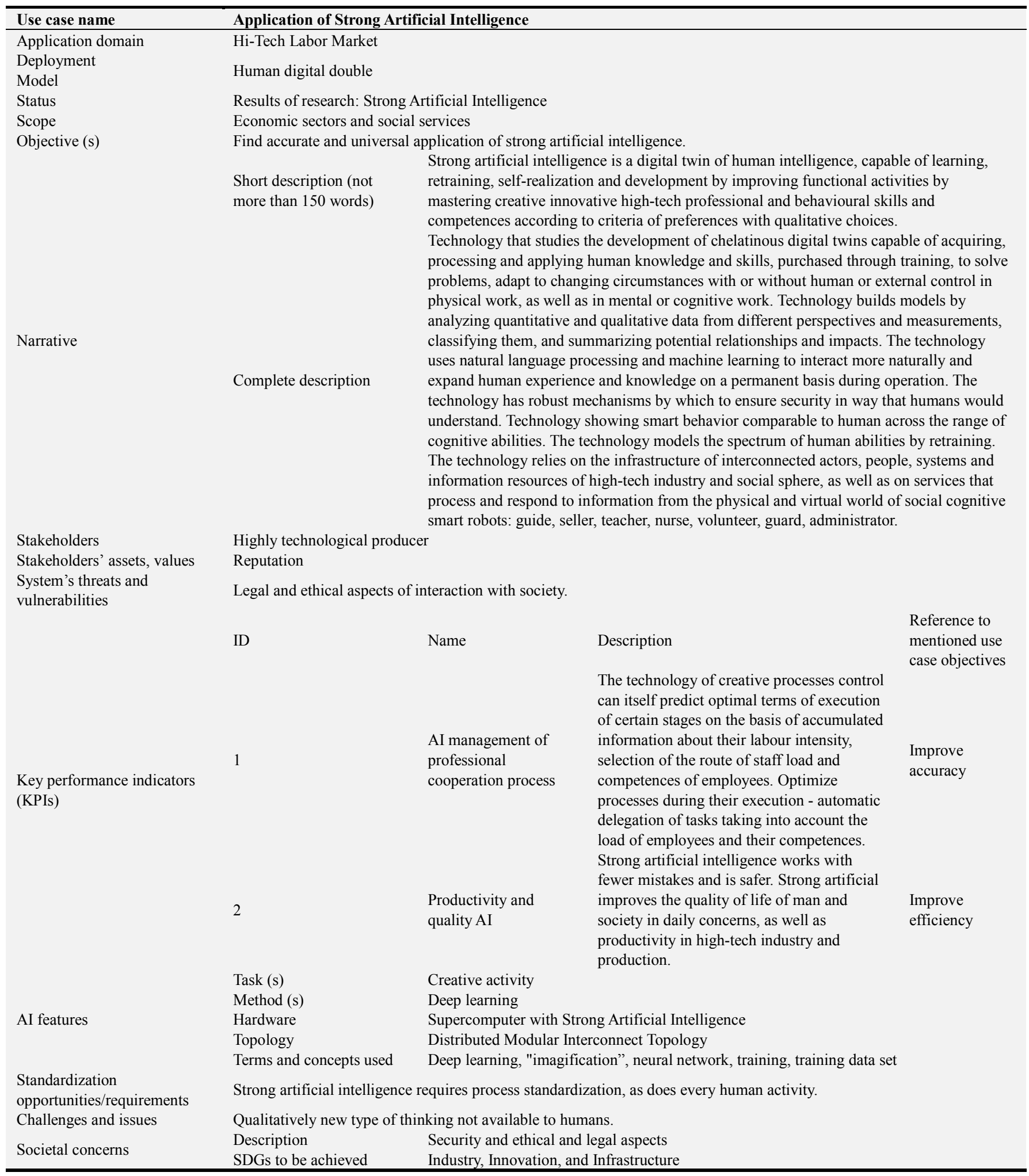

Table 2. Data.

\begin{tabular}{ll}
\hline Data characteristics & \\
\hline Description & Strong Artificial Intelligence Data \\
Source & Model and technology of Strong Artificial Intelligence \\
Type & Strong \\
Volume (size) & Hi-Tech Labor Market \\
\hline
\end{tabular}




\begin{tabular}{ll}
\hline Data characteristics & \\
\hline Velocity (e.g. real time) & Supercomputering Velocity \\
Variety (multiple datasets) & streams of multiple datasets \\
Variability (rate of change) & Retraining \\
Quality & High \\
\hline
\end{tabular}

Table 3. Process scenario.

\begin{tabular}{|c|c|c|c|c|c|}
\hline \multicolumn{6}{|c|}{ Scenario conditions } \\
\hline $\mathbf{N}$. & Scenario name & Scenario description & Triggering event & Pre-condition & Post-condition \\
\hline 1 & Training & $\begin{array}{l}\text { Train a model (deep neural } \\
\text { network) with training data set }\end{array}$ & $\begin{array}{l}\text { Technological process raw } \\
\text { data set is ready }\end{array}$ & Formatting of data & Management of safety \\
\hline 2 & Evaluation & Expansion of the trained model & $\begin{array}{l}\text { Development of } \\
\text { technological thinking and } \\
\text { behaviour }\end{array}$ & $\begin{array}{l}\text { Cognitive thinking patterns } \\
\text { and psychological } \\
\text { behaviors }\end{array}$ & $\begin{array}{l}\text { Meeting KPI requirements } \\
\text { is condition of } \\
\text { development }\end{array}$ \\
\hline 3 & Execution & Model and Technology Tooling & Interaction & Activization of Model & Completion of interaction \\
\hline 4 & Retraining & $\begin{array}{l}\text { Retrain a model with training data } \\
\text { set }\end{array}$ & $\begin{array}{l}\text { Certain period of time has } \\
\text { passed since the last } \\
\text { training/ retraining }\end{array}$ & $\begin{array}{l}\text { Additional data and } \\
\text { knowledge }\end{array}$ & $\begin{array}{l}\text { Combining Data and } \\
\text { Knowledge }\end{array}$ \\
\hline
\end{tabular}

Table 4. Training.

\begin{tabular}{|c|c|c|c|c|c|}
\hline Scenario name & Training & & & & \\
\hline Step No. & Event & Name of process/Activity & Primary actor & Description of process/activity & Requirement \\
\hline 1 & $\begin{array}{l}\text { Sample raw data set is } \\
\text { ready }\end{array}$ & $\begin{array}{l}\text { Specification and } \\
\text { classification }\end{array}$ & Manufacturer & Transform sample raw data & $\begin{array}{l}\text { Strong AI } \\
\text { Software }\end{array}$ \\
\hline 2 & Completion of Step 1 & $\begin{array}{l}\text { Creating Set of } \\
\text { Experimental Data }\end{array}$ & Manufacturer & $\begin{array}{l}\text { Development of set of experimental } \\
\text { data through job modelling }\end{array}$ & $\begin{array}{l}\text { Software of } \\
\text { modelling }\end{array}$ \\
\hline 3 & Completion of Step 2 & Model training & $\begin{array}{l}\text { AI solution } \\
\text { provider }\end{array}$ & $\begin{array}{l}\text { Train a model (deep neural } \\
\text { network) with experimental data set } \\
\text { created by Step } 2\end{array}$ & Big Data \\
\hline
\end{tabular}

Table 5. Evaluation

\begin{tabular}{|c|c|c|c|c|c|}
\hline Scenario name & Evaluation & & & & \\
\hline Step No. & Event & Name of process/Activity & Primary actor & Description of process/activity & Requirement \\
\hline 1 & $\begin{array}{l}\text { Completion of } \\
\text { training/retraining }\end{array}$ & Research & Manufacturer & $\begin{array}{l}\text { Train model (deep neural network) with } \\
\text { experimental data set created }\end{array}$ & Big Data \\
\hline 2 & $\begin{array}{l}\text { Completion of } \\
\text { Step } 1\end{array}$ & Identification & AI solution provider & $\begin{array}{l}\text { Based on data, detect execution using a } \\
\text { deep neural network trained in learning } \\
\text { scenario }\end{array}$ & Smart Data \\
\hline 3 & $\begin{array}{l}\text { Completion of } \\
\text { Step } 2\end{array}$ & Evaluation & Manufacturer & $\begin{array}{l}\text { Comparison of phase } 2 \text { results with } \\
\text { human performance }\end{array}$ & $\begin{array}{l}\text { Efficiency } \\
\text { and quality }\end{array}$ \\
\hline \multicolumn{2}{|c|}{ Input of evaluation } & $\begin{array}{l}\text { Productivity } \\
\text { Efficiency and quality }\end{array}$ & & & \\
\hline
\end{tabular}

Table 6. Execution

\begin{tabular}{|c|c|c|c|c|c|}
\hline Scenario name & Execution & & & & \\
\hline Step No. & Event & $\begin{array}{l}\text { Name of } \\
\text { process/Activity }\end{array}$ & $\begin{array}{l}\text { Primary } \\
\text { actor }\end{array}$ & Description of process/activity & Requirement \\
\hline 1 & $\begin{array}{l}\text { Completion of comparison of } \\
\text { modeling results with human } \\
\text { performance }\end{array}$ & Research & Manufacturer & $\begin{array}{l}\text { Development of a set of experimental } \\
\text { data through job modelling }\end{array}$ & Quality \\
\hline 2 & Completion of Step 1 & Identification & Manufacturer & $\begin{array}{l}\text { Based on modified data train model } \\
\text { (deep neural network) with } \\
\text { experimental data set created }\end{array}$ & Compatibility \\
\hline \multicolumn{2}{|c|}{ Input of Execution } & Modification & & & \\
\hline \multicolumn{2}{|c|}{ Output of Execution } & Compatibility & & & \\
\hline
\end{tabular}

Table 7. Retraining.

\begin{tabular}{|c|c|c|c|c|c|}
\hline Scenario name & Retraining & & & & \\
\hline Step No. & Event & $\begin{array}{l}\text { Name of } \\
\text { process/ } \\
\text { Activity }\end{array}$ & Primary actor & Description of process/activity & Requirement \\
\hline 1 & $\begin{array}{l}\text { Certain period of time has } \\
\text { passed since the last } \\
\text { training/retraining }\end{array}$ & Research & Manufacturer & Additional data and knowledge & Completeness \\
\hline
\end{tabular}




\begin{tabular}{|c|c|c|c|c|c|}
\hline Scenario name & Retraining & & & & \\
\hline Step No. & Event & $\begin{array}{l}\text { Name of } \\
\text { process/ } \\
\text { Activity }\end{array}$ & Primary actor & Description of process/activity & Requirement \\
\hline 2 & Completion of Step 1 & $\begin{array}{l}\text { Experimental } \\
\text { data set creation }\end{array}$ & Manufacturer & $\begin{array}{l}\text { Combining Data and Knowledge } \\
\text { Based on modified data train model } \\
\text { (deep neural network) with experimental } \\
\text { data set created }\end{array}$ & Compatibility \\
\hline 3 & Completion of Step 2 & Model training & $\begin{array}{l}\text { AI solution } \\
\text { provider }\end{array}$ & $\begin{array}{l}\text { Comparison of phase } 2 \text { results with } \\
\text { human performance }\end{array}$ & $\begin{array}{l}\text { Efficiency and } \\
\text { quality }\end{array}$ \\
\hline \multicolumn{2}{|c|}{ Specification of retraining data } & \multicolumn{4}{|c|}{ Retraining data set has to include recent data } \\
\hline
\end{tabular}

\section{Conclusion}

There are a number of social problems associated with human-machine interaction. Sophisticated artificial intelligence technologies are not yet credible by citizens. The coming years will take to improve safety and standardize the creation and application of creative creative life-saving strong artificial intelligence. International standardization of the production and use of intelligent agents ensuring their compatibility has intensified. Intelligent agents can be combined at the software level through a standard module of communication with them. Compatibility will allow produce from them intelligent systems with cognitive creative and behavioral abilities of the person for service services. It will also allow to produce intelligent production high-tech complexes. Standardization of creative viable strong artificial intelligence will help to ensure the interface, compatibility and synergy of its safe application in various sectors of economy, industry and service. Developing approaches to creative lifesaving strong artificial intelligence technologies that are beneficial to humanity remains one of the most pressing challenges. Its solution may be an incentive for a technology revolution for sustainable development. In the future, reliable and safe creative life-saving strong artificial intelligence will significantly change the life of the world community [20].

\section{References}

[1] Evgeniy Bryndin. Cognitive Robots with Imitative Thinking for Digital Libraries, Banks, Universities and Smart Factories. International Journal of Management and Fuzzy Systems. V. 3, N. 5, 2017, pp 57- 66 .

[2] Evgeniy Bryndin. Technological Thinking, Communication and Behavior of Androids. Communications. Vol. 6, No. 1, 2018. Pages: 13-19.

[3] Venture radar - "Top Service Robotics Companies", 2018. URL:

https://www.ventureradar.com/keyword/Service\%20Robotics.

[4] Pedro Domingos. Supreme algorithm. How machine learning will change our world. - M.: Mann, Ivanov and Ferber, 2016. -336 pages.

[5] Evgeniy Bryndin. Program Hierarchical Realization of Adaptation Behavior of the Cognitive Mobile Robot with Imitative Thinking. International Journal of Engineering Management. Volume 1, Issue 4. 2017, pp. 74-79.
[6] Evgeniy Bryndin. Social Cognitive Smart Robots: Guide, Seller, Lecturer, Vacuum Cleaner, Nurse, Volunteer, Security Guard, Administrator. Communications. Volume 7, Issue 1. 2019. Pages: 6-12.

[7] Report of COMEST on robotics ethics, 2017. URL: https://unesdoc.unesco.org/ark:/48223/pf0000253952he.

[8] Evgeniy Bryndin. Technological, Economic and Social Aspects of Management by Development of the Digital Industry 4.0. International Journal of Managerial Studies and Research (IJMSR), vol 6, no. 4, 2018, pp. 19-30.

[9] Evgeniy Bryndin. Directions of Development of Industry 4.0, Digital Technology and Social Economy. American Journal of Information Science and Technology. Volume 2, Issue 1. 2018. P. 9-17.

[10] Evgeniy Bryndin. Human Digital Doubles with Technological Cognitive Thinking and Adaptive Behaviour. Software Engineering, Volume 7, Issue 1, 2019. P. 1-9.

[11] Evgeniy Bryndin. System retraining to professional competences of cognitive robots on basis of communicative associative logic of technological thinking. International Robotics Automation Journal. 2019; 5 (3): 112-119.

[12] Artificial Intelligence \& Robotics: Industry Report \& Investment Case. 2019.

https://indexes.nasdaqomx.com/docs/NQROBO\%20Research.pdf.

[13] Deep Learning based Recommender System: A Survey and New Perspectives, Shuai Zhang et al., 2018. https://arxiv.org/pdf/1707.07435.pdf.

[14] Evgeniy Bryndin. Robots with Artificial Intelligence and Spectroscopic Sight in Hi-Tech Labor Market. International Journal of Systems Science and Applied Mathematic, Vol. 4, Issue 1, 2019.

[15] Google's Artificial Intelligence Built an AI That Outperforms Any Made by Humans. 2019 https://futur-ism.com/googleartificial-intelligence-built-ai.

[16] Evgeniy Bryndin. Supercomputer BEG with Artificial Intelligence of Optimal Resource Use and Management by Continuous Processing of Large Programs. International Journal of Research in Engineering, Vol. 1, Issue 2, 2019. Pages: 9-14.

[17] Evgeniy Bryndin. Robots with Artificial Intelligence and Spectroscopic Sight in Hi-Tech Labor Market. International Journal of Systems Science and Applied Mathematic, V. 4, № 3, 2019. Pages: 31-37.

[18] Evgeniy G. Bryndin. Modeling of Transformation of Nanostructures by Cognitive Systems on the Basis of Big Smart Data. International Journal of Artificial Intelligence and Mechatronics. Volume 7, Issue 4. 2019. P. 19-22. 
[19] Evgeniy Bryndin Formation Smart Data Science for Automated Analytics of Modeling of Scientific Experiments. American Journal of Software Engineering and Applications. Volume 8, Issue 2, 2019. Pages: 36-43.
[20] Evgeniy Bryndin. Creative innovative transformational ecosystem of formation of humane technological society. International Robotics Automation Journal. 2019; 5 (3): 91-94. 